\title{
Avaliação epidemiológica da sífilis congênita na região Nordeste do Brasil
}

\author{
Epidemiological evaluation of congenital syphilis in northeastern Brazil \\ Evaluación epidemiológica de la sífilis congénita en el noreste de Brasil
}

Recebido: 28/12/2020 | Revisado: 06/01/2021 | Aceito: 14/03/2021 | Publicado: 21/03/2021

\author{
Evaldo Hipólito de Oliveira \\ ORCID: https://orcid.org/0000-0003-4180-012X \\ Universidade Federal do Piauí, Brasil \\ E-mail: evaldohipólito@gmail.com \\ Elison Costa Holanda \\ ORCID: https://orcid.org/0000-0001-9130-7873 \\ Universidade Federal do Piauí, Brasil \\ E-mail: holandap2@outlook.com \\ Larissa Conceição da Silva \\ ORCID: https://orcid.org/0000-0002-0173-1513 \\ Universidade Federal do Piaú, Brasil \\ E-mail: larissafarmaufpi@oulook.com \\ Maria Carolina de Sousa Brito \\ ORCID: https://orcid.org/0000-0001-5633-7372 \\ Universidade Federal do Piaú, Brasil \\ E-mail: carol.sousab@hotmail.com \\ Patrícia Caroline Machado de Sousa \\ ORCID: https://orcid.org/0000-0002-0866-7235 \\ Universidade Federal do Piaú, Brasil \\ E-mail: patricia_caroline4@outlook.com
}

\begin{abstract}
Resumo
A sífilis é uma Infecção Sexualmente Transmissível causada pelo Treponema Pallidum, na qual o tratamento da mesma durante a gestação é indispensável, contudo, se não tratada pode evoluir para quadros de neonatos precoces e a morte fetal. A sífilis congênita é uma patologia de notificação compulsória, categorizada como doença infectocontagiosa devido a propagação do T. pallidum por via transplacentária, durante a gravidez. No Brasil, os dados do boletim de 2018 do Ministério da Saúde foram notificados 158.051 casos de sífilis onde 26.219 dos casos representavam sífilis congênita evoluindo para 241 óbitos. Este artigo buscou descrever o perfil dos casos de sífilis na gestação, notificados na região Nordeste, Brasil, do ano de 2009 ao 2018. Foi realizado um levantamento epidemiológico de sífilis congênitas na região Nordeste no período de 2009 a 2018. Os resultados obtidos através do levantamento demonstram aumento da incidência no diagnóstico por ano a cada mil nascidos vivos, tal como observa-se para gestantes de 20 a 29 anos, bem como no diagnóstico durante o segundo e terceiro trimestre da gestação, além de apresentar um número crescente nos casos de adesão ao tratamento, retratando número crescente de mães que realizaram o pré-natal. A prevalência de sífilis foi observada em mulheres com nível de escolaridade mais baixos. Em decorrência desse levantamento de dados, viu-se a relevância que o estudo apresenta, tendo em vista a importância epidemiológica destes dados para a aplicação de políticas públicas.
\end{abstract}

Palavras-chave: Infecção sexualmente transmissível; Sífilis na gestação; Saúde pública.

\begin{abstract}
Syphilis is a sexually transmitted infection caused by Treponema Pallidum, where treatment during pregnancy is indispensable, but if left untreated it can progress to early neonates and fetal death. A congenital infection is a condition of compulsory notification, categorized as infectious disease due to the spread of T. pallidum transplacentally during pregnancy. In Brazil, data from the Ministry of Health bulletin 2018 were reported 158,051 case cases where 26,219 cases represent cases of evolutionary conflict for 241 cases. This article aimed to describe the profile of pregnancy cases, reported in the Northeast region, Brazil, from 2009 to 2018. An epidemiological survey of congenital individuals in the Northeast region from 2009 to 2018 was performed. Survey demonstrated increase in diagnosis per year every thousand live births, as observed in pregnant women aged 20 to 29 years, as well as in the diagnosis during the second and third trimester of pregnancy, in addition to showing an increasing number in cases of adherence to treatment, portraying the increasing number of mothers who had or pre-performed -Christmas. The prevalence of syphilis was observed in women with lower education level. As a result of the data collection, you can see the relevance of the study presented, considering the epidemiological importance of these data for the application of public policies.
\end{abstract}

Keywords: Sexually transmitted infection; Syphilis in pregnancy; Public health. 


\begin{abstract}
Resumen
La sífilis es una infección de transmisión sexual causada por Treponema pallidum, en la cual su tratamiento durante el embarazo es indispensable, sin embargo, si no se trata puede evolucionar a recién nacidos precoces y muerte fetal. La sífilis congénita es una patología de notificación obligatoria, categorizada como una enfermedad infecciosa-contagiosa por la propagación de T. pallidum por vía transplacentaria, durante el embarazo. En Brasil, los datos del boletín del Ministerio de Salud de 2018 reportaron 158,051 casos de sífilis donde 26,219 de los casos representaron sífilis congénita que evolucionaron a 241 muertes. Este artículo buscó describir el perfil de los casos de sífilis durante el embarazo, reportados en la región Nordeste, Brasil, de 2009 a 2018. Se realizó una encuesta epidemiológica de sífilis congénita en la región Nordeste de 2009 a 2018. Los resultados obtenidos a través de la encuesta muestran un aumento en la incidencia de diagnóstico por año por mil nacidos vivos, como se observa en mujeres embarazadas de 20 a 29 años, así como en el diagnóstico durante el segundo y tercer trimestre de gestación, además de un número creciente en la adherencia al tratamiento, retratando un número cada vez mayor de madres que se sometieron a cuidados prenatales. La prevalencia de sífilis se observó en mujeres con menor nivel educativo. Como resultado de esta recolección de datos, se vio la relevancia que presenta el estudio, dada la importancia epidemiológica de estos datos para la aplicación de políticas públicas.
\end{abstract}

Palabras clave: Infección de transmisión sexual; Sífilis en el embarazo; Salud pública.

\title{
1. Introdução
}

Durante a gestação adquirir a garantia do acompanhamento pré-natal é um importante meio para atenuar os riscos de transmissão vertical da sífilis (Macêdo et al., 2009). A sífilis é uma Infecção Sexualmente Transmissível causada pelo Treponema Pallidum, na qual o tratamento da mesma durante a gestação é indispensável, contudo, se não tratada pode evoluir para quadros de neonatos precoces e a morte fetal (Saraceni et al., 2017). Desse modo, a sífilis congênita demonstra um risco epidemiológico alto por ser uma das principais causas de morbidade intra-uterina, fazendo-se responsável por $50 \%$ dos dados negativos relacionados ao feto (Macêdo et al., 2009).

De acordo com a Organização Mundial da Saúde em 2016, os casos de sífilis congênita representavam o valor de 661 mil casos, com aproximadamente 200 mil natimortos e mortes neonatais. No Brasil, a realidade não é distante, uma vez que em dados do boletim de 2018 do Ministério da Saúde foram notificados 158.051 casos de sífilis onde 26.219 dos casos representavam sífilis congênita evoluindo para 241 óbitos. Vislumbrando o alcance das metas em 1997 o Ministério da Saúde considerou como parâmetro, a elisão de um caso por mil nascidos vivos, todavia, este padrão foi inatingível mantendo a patologia como ponto crucial a ser articulado, uma vez que o número de subnotificações é considerável e de acordo com Araújo et al., (2012), alguns estudos realizados em hospitais inferem que a incidência de casos seria de 9,9 a 22 por mil nascidos vivos.

A sífilis congênita é uma patologia de notificação compulsória, destacando a obrigatoriedade no Brasil desde 1986, categorizada como doença infectocontagiosa devido a propagação do T. pallidum por via transplacentária, durante a gravidez. Dessa forma o agravo resultará em consequências de alto grau, como surdez neurológica, ceratite intestinal com cegueira, hidrocefalia e retardo mental, no entanto o grau da sequela decorrente é diversificado levando-se em considerações alguns quesitos como, tempo de exposição fetal ao treponema, a carga treponêmica materna, o tratamento da infecção da mãe e a virulência (De França et al., 2015). No Nordeste, tem-se a necessidade de uma atenção voltada a esta área, uma vez que, de acordo com os dados do Boletim epidemiológico de 2018, dentre os 11 estados que se encontram acima da média nacional a cada 100.000 portadores de sífilis congênita 5 deles são estados do Nordeste. Dessa forma, o presente estudo tem como objetivo traçar o perfil epidemiológico da sífilis congênita na região Nordeste.

\section{Metodologia}

Para realização de estudos científicos é necessário o uso de métodos e técnicas que melhor articulem o andamento da pesquisa, contudo existem formas documentais em institutos de pesquisa, órgãos públicos e departamentos de registro e estatística (Oliveira et al., 2019).

Trata-se de um estudo epidemiológico, retrospectivo e descritivo de cunho populacional, utilizando-se dados 
secundários, no período de 2009 até 2018 referente a região Nordeste. Os dados foram coletados no site do Departamento de Informática do Sistema Único de Saúde (DATASUS), a partir do Sistema de Informação de Agravos de Notificação (SINAN). As informações estão na seção de informação de saúde (TABNET), na opção epidemiologia e morbidades. Devido ao estudo ter utilizado somente dados secundários de domínio público disponibilizados pelo Ministério da Saúde através do DATASUS não houve necessidade de submeter essa pesquisa ao Comitê de Ética de acordo com a Resolução CNS 466/12.

O programa Microsoft ${ }^{\circledR}$ Excel foi utilizado para organização dos dados. As variáveis de estudos utilizadas foram: raça, faixa etária, escolaridade. Os dados foram colocados em gráficos elaborados a partir do programa Microsoft Office- Excel.

\section{Resultados e Discussão}

A sífilis congênita é o modo de transmissão de maior impacto para a saúde pública devido à alta frequência com que produz desfechos graves para a gestação e para a criança, a exemplo de parto prematuro, óbito fetal e neonatal e infecção congênita do recém-nascido. (Brasil, 2006).

Pode-se visualizar o aumento do número de casos registrados de sífilis congênita no Brasil durante do ano de 2009 até 2018 onde no ano de 2009 encontra-se um total de 2000 e em 201816000 revelando um aumento de oito vezes maior que 2009 (Gráfico 1), pode ser atribuído a diminuição do número de sub registros e consequentemente o aumento de notificação, bem como ações de vigilância epidemiológicas direcionadas para uma melhor abordagem e identificação dos casos de doença (Lima et al., 2017). Do mesmo modo, evidencia as deficiências dos serviços de saúde, particularmente da atenção ao pré-natal, pois o diagnóstico precoce e tratamento da gestante são medidas relativamente simples e bastante eficazes na prevenção dessa forma da doença (Holztrattne et al., 2019).

Gráfico 1: Número de casos e taxa de detecção a cada mil nascidos vivos de gestantes com sífilis por ano de diagnóstico no Nordeste durante o período de 2009-2018.

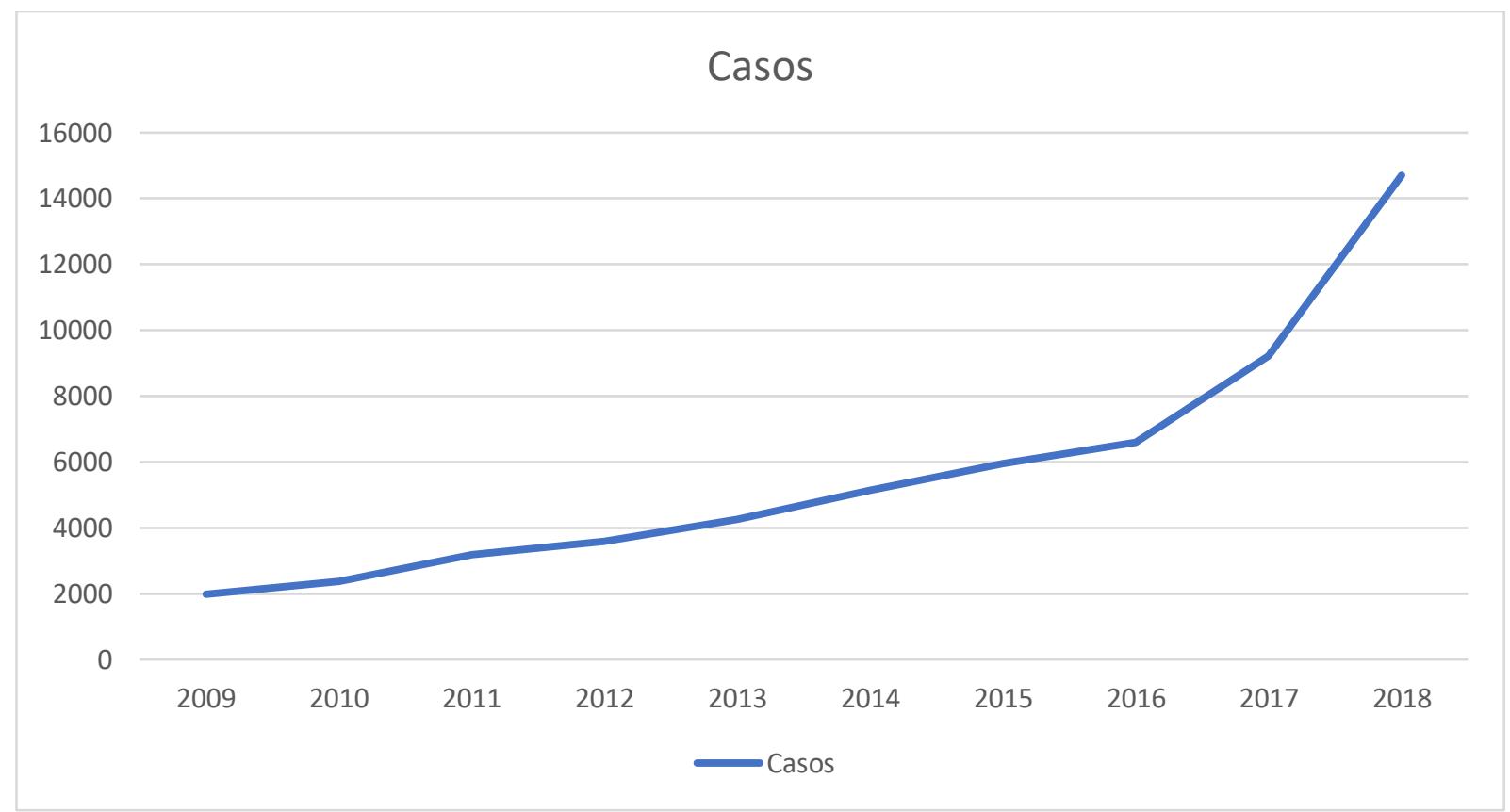

Fonte: Ministério da Saúde/SVS - Sistema de Informação de Agravos de Notificação - SINAN Net. (2018).

Quanto ao número de casos de acordo com a idade gestacional é possível inferir que o número de gestantes com diagnóstico no terceiro trimestres da gestação foi superior ao primeiro e ao segundo trimestre (Gráfico 2), apesar de que a grande maioria das mulheres teve a sífilis diagnosticada no período pré-natal, a ocorrência revela ser muito provável que a assistência 
não tenha sido de qualidade. É possível que, mesmo quando o diagnóstico ocorreu no pré-natal, grande parte se deu em um período tardio, considerando que a maioria das notificações ocorreu entre o segundo e terceiros trimestres de gestação. No Brasil houve melhora nos indicadores de cobertura de pré-natal, entretanto não têm sido suficientes para garantir assistência de qualidade. Estudos mostram também a importância da assistência pré-natal de qualidade com diagnóstico precoce da sífilis nas gestantes e destacam as consequências do tratamento inadequado da mesma sobre a morbimortalidade das crianças (Cardoso $e t$ al., 2018).

Gráfico 2: Número de casos de gestantes com sífilis segundo idade gestacional em cada ano no período de2009-2018 de acordo com o trimestre da gestação.

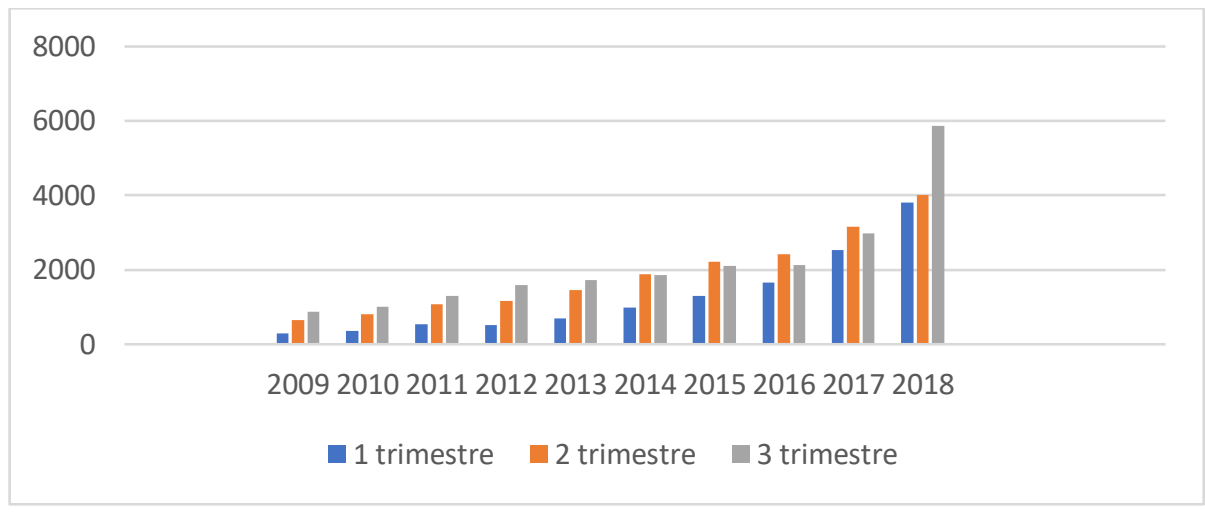

Fonte: Ministério da Saúde/SVS - Sistema de Informação de Agravos de Notificação - SINAN Net. (2018).

Em relação ao número de casos de mães que possuem diagnóstico de sífilis e realizaram o pré-natal pode-se observar um número significativo de mães que realizaram o pré-natal dentre os anos de 2009 e 2017, demonstrando-se crescente ao longo desse período (Gráfico 3). O Ministério da Saúde, como medida para reduzir as altas taxas de mortalidade materna, estabeleceu recomendações como: realizar até o quarto mês de gestação a primeira consulta ao pré-natal, ter no mínimo seis consultas até o fim do pré-natal, implementar sistemas de esclarecimentos de dúvidas e informações, atividades educativas e estimular a realização de parto normal. Portanto, garantir com que a gestante tenha um pré-natal adequado permite prevenir, diagnosticar e tratar acontecimentos indesejáveis na gestação (Pires et al., 2014).

No entanto há alguns fatores limitantes para a realização desses procedimentos destacando aspectos que impulsionam a falha para atingir metas estabelecidas para eliminar ou atenuar a patologia são destacados como, dificuldade de atendimento dos serviços de saúde, monitoramento dos parceiros sexuais das gestantes com positividade, inexistência na solicitação para realizar exame sorológico das gestantes (De França et al., 2015). Diante disso, Da Costa, (2012) afirma que essa problemática, poderia ser evitada com uma boa qualidade de atendimento ao pré-natal, onde seriam superados todos esses fatores. 
Gráfico 3: Número de casos de sífilis congênita segundo informação sobre realização de pré-natal da mãe por ano de diagnóstico no Nordeste no período de 2009-2017.

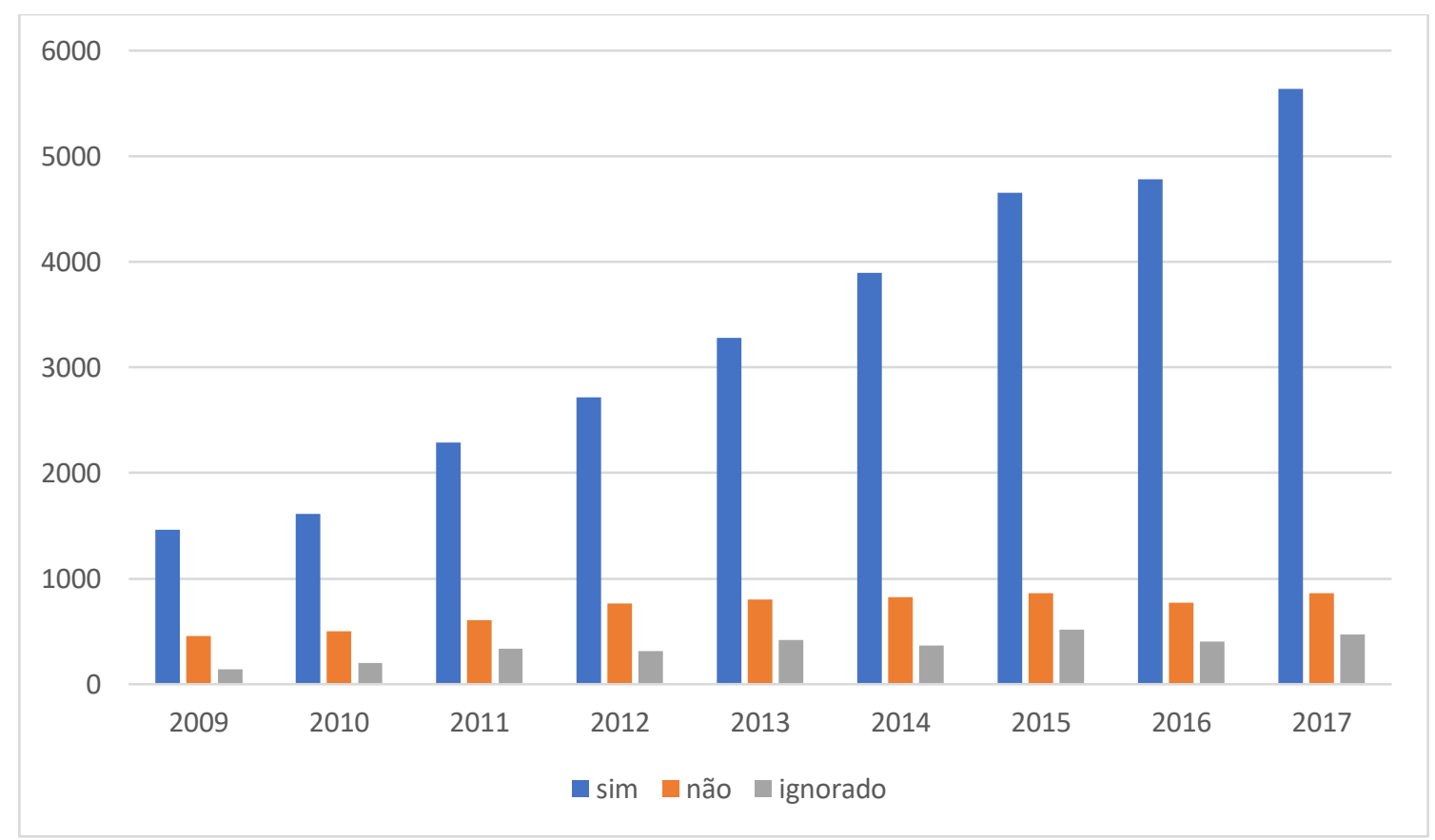

Fonte: Ministério da Saúde/SVS - Sistema de Informação de Agravos de Notificação - SINAN Net. (2018).

É possível identificar a prevalência de sífilis congênita entre mulheres que possuem o ensino fundamental incompleto ao passo que mulheres que possuem superior incompleto e completo demonstram quantidade inexistente de diagnóstico para sífilis (Gráfico 4). Visto que está relacionado a condições socioeconômicas de renda e escolaridade Domingues et al., (2016), afirma que a escolaridade estar relacionada com a incidência, pois em seu estudo concluiu que quanto menor a escolaridade da mulher, maior a infecção por sífilis e sífilis congênita.

Gráfico 4: Número de casos de sífilis congênita segundo a escolaridade da mãe por ano de diagnóstico no Nordeste durante período de 2009-2018.

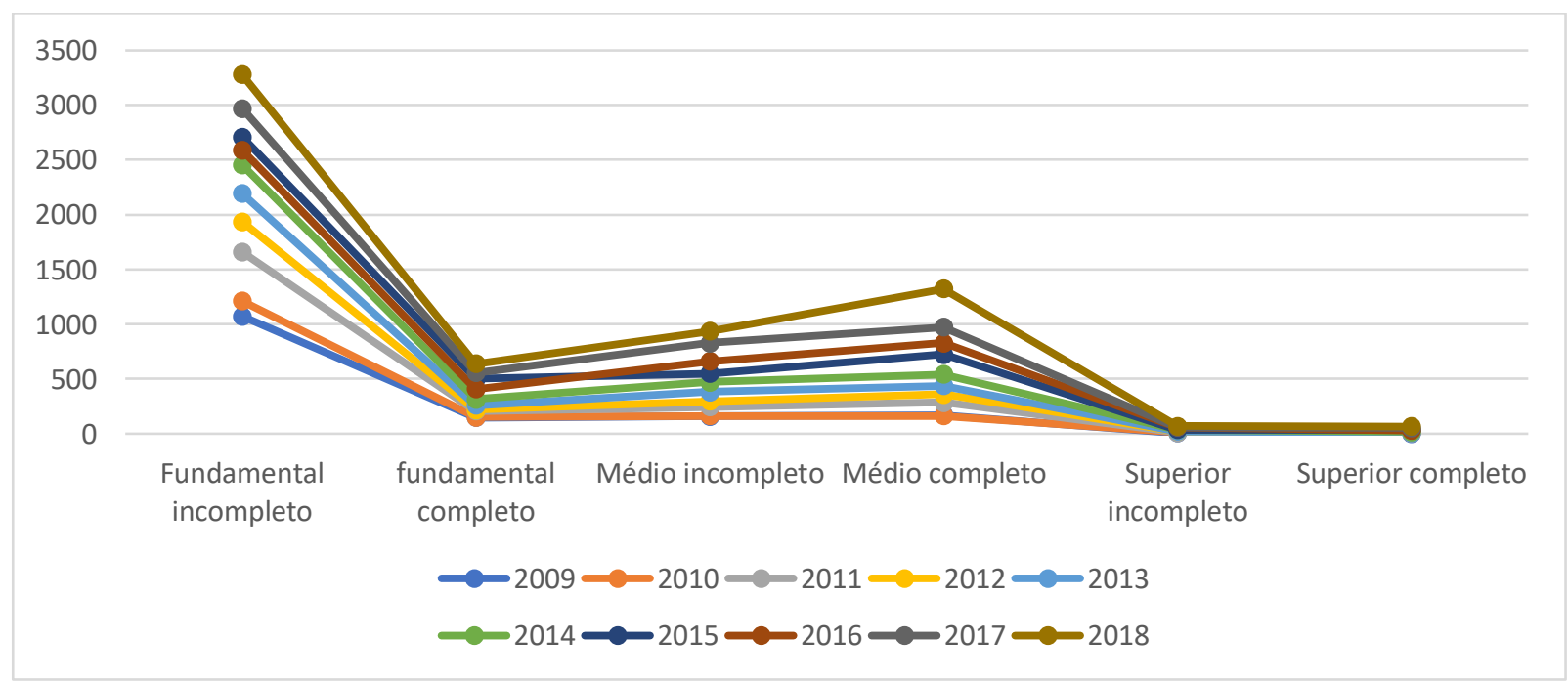

Fonte: Ministério da Saúde/SVS - Sistema de Informação de Agravos de Notificação - SINAN Net. (2018). 
Vale ressaltar que houve uma predominância de jovens de 20 a 29 ano durante o período de 2007 a 2018 demonstrandose mais acentuado no ano de 2018 com 14.000 casos (Gráfico 5), dado que destaca a importância de se desenvolver trabalhos de prevenção e promoção da saúde junto a essa parcela da população, além de incluir a realização do teste rápido (TR) em qualquer oportunidade de atendimento à mulher, bem como dos seus parceiros sexuais, independentemente do motivo da procura pelo serviço de saúde. O TR é um recurso de alto custo-efetividade e fácil execução, tornando-se assim uma ferramenta valorosa no diagnóstico e tratamento precoce da gestante juvenil e, consequentemente, no combate à sífilis congênita (Cardoso et al., 2018).

Os fatores sociodemográficos são marcantes nessa sendo necessário conhecer esses aspectos e como podem afetar, no qual segundo Cavalcante et al., (2019), uma baixa condição socioeconômica pode dificultar o comparecimento das crianças aos postos de saúde, inferindo que filhos de mães solteiras retornam menos ao serviço de saúde, correlacionando ao fato de serem as responsáveis pelo lar, apresentando maior objeção para se ausentar do trabalho, além disso há também a falta de renda.

Gráfico 5: Número de caso de gestantes com sífilis congênita segundo faixa etária por ano de diagnóstico. Nordeste, 20092018.

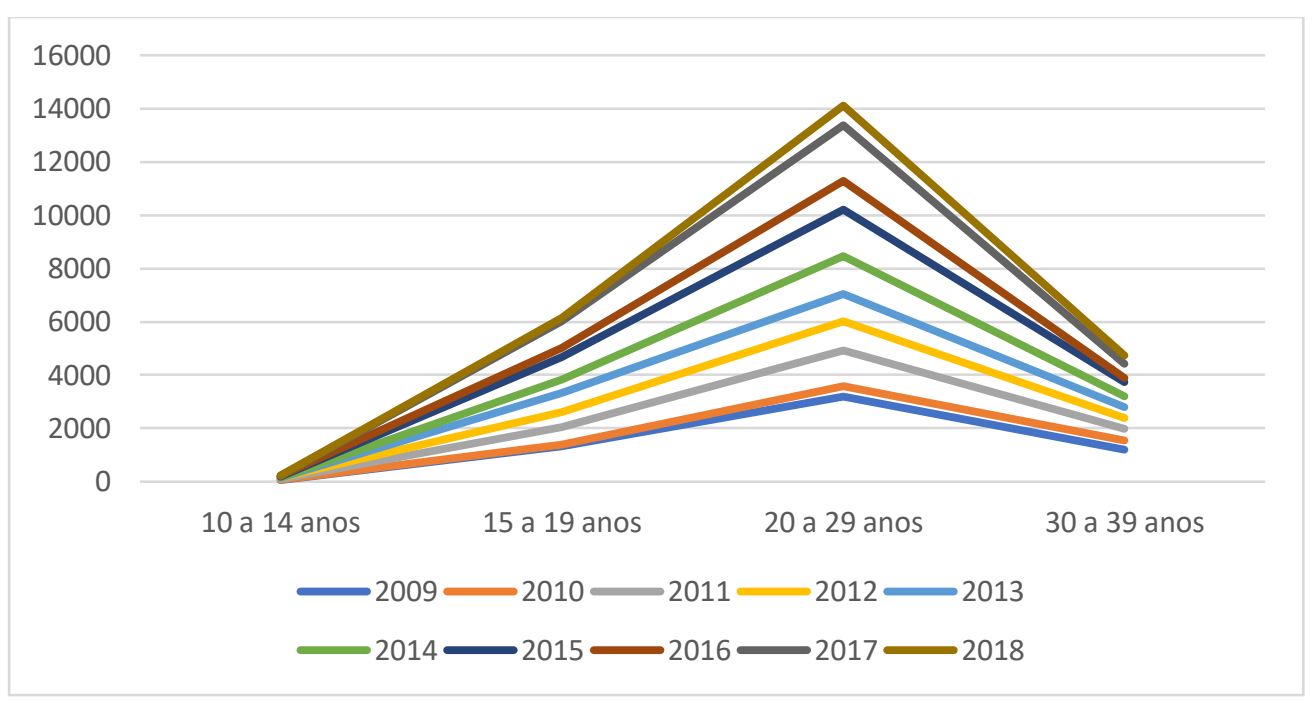

Fonte: Ministério da Saúde/SVS - Sistema de Informação de Agravos de Notificação - SINAN Net. (2018).

Pode-se notar que o número de casos de sífilis congênita foi predominante em gestantes que tiveram o diagnóstico de sífilis materna no período em que realizaram o pré-natal, no entanto também se destaca um número elevado durante o momento do parto/curetagem observando que 2018 em relação a 2019 houve redução desses casos (Gráfico 6).Na maioria dos casos o diagnóstico foi feito durante esse acompanhamento, entretanto, um tratamento adequado ou a falta dele poderiam explicar a transmissão vertical da sífilis. Um dado de alta relevância seria da efetividade e qualidade da realização da consulta de pré-natal, no que se diz respeito ao início precoce, número adequado de consultas e disponibilidade de exames para a testagem de sífilis e outras doenças (Vescovi \& Schuelter-Trevisol, 2020) 
Gráfico 6: Número de casos de sífilis congênita segundo o momento do diagnóstico da sífilis materna por ano de diagnóstico no Nordeste durante o período de 2009-2018.

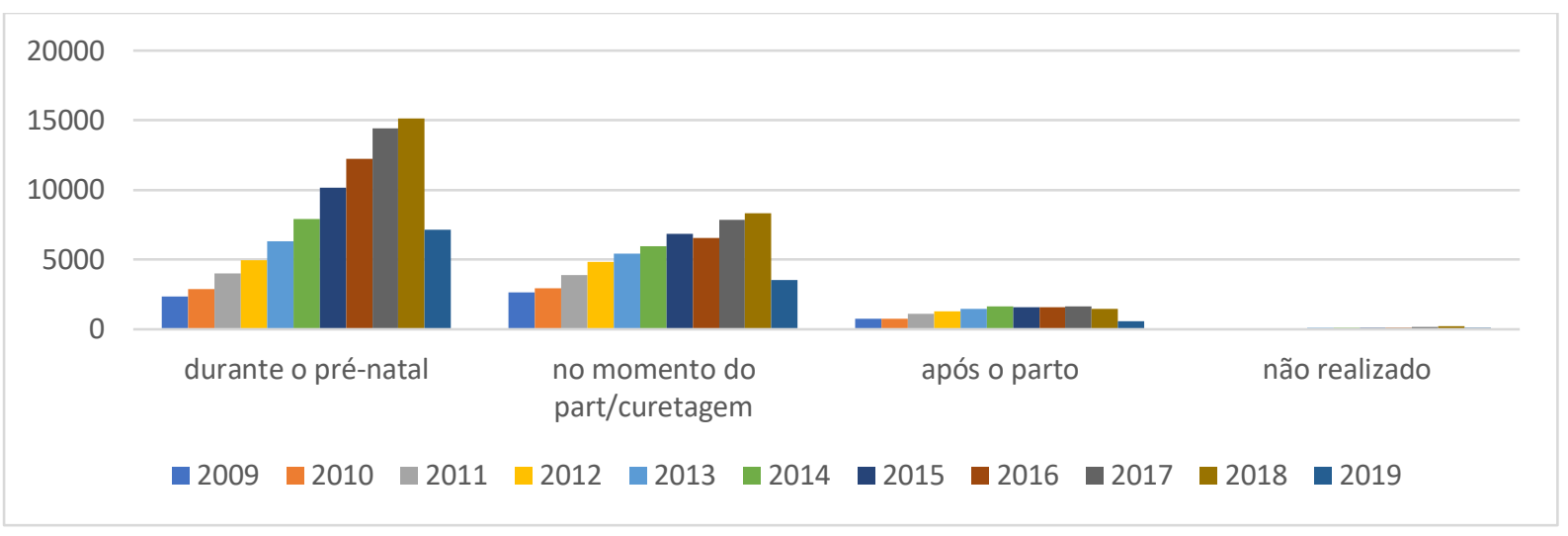

Fonte: Ministério da Saúde/SVS - Sistema de Informação de Agravos de Notificação - SINAN Net. (2018).

\section{Considerações Finais}

De acordo com os resultados obtidos podemos observar que houve um aumento do número de casos registrados de sífilis congênita no Nordeste. Tal fato decorre do crescente número de registros e notificações como também de uma melhor identificação dos casos de doença. Além disso, pode-se destacar que a escolaridade é um ponto relevante no que diz respeito a Sífilis congênita, ou seja, onde há um menor acesso educação, percebe-se um maior número de casos.

Porém, apesar da identificação da doença, muitas pacientes não receberam assistência de qualidade suficientes para um pré-natal adequado. Em decorrência do levantamento de dados, viu-se a relevância que o estudo apresenta, tendo em vista a importância epidemiológica destes dados para a aplicação de políticas públicas.

Como perspectivas futuras vislumbramos melhorar as políticas públicas na região nordestina, por meio de maior planejamento geográfico, com construção de mapas que destaquem as regiões mais críticas para um controle mais efetivo, bem como analisar outras IST e patologias prevalentes, nas quais podem ser controladas a partir de melhorias na saúde pública.

\section{Referências}

Araújo, C. L., Shimizu, H. E., Sousa, A. I. A., \&Hamann, E. M. (2012). Incidência da sífilis congênita no Brasil e sua relação com a Estratégia Saúde da Família. Revista de Saúde Pública, 46(3), 479-486. https://doi.org/10.1590/S0034-89102012000300010

Brasil, Ministério da Saúde (MS). (2006). Programa Nacional de Doenças Sexualmente Transmissíveis e AIDS. Diretrizes para controle da sífilis congênita: manual de bolso. Brasília: MS.

Brasil, Ministério da Saúde. (2016). Ministério da Saúde lança ação nacional de combate à sífilis. Disponível em: <http://portalms.saude.gov.br/noticias/agenciasaude/26100-ministerio-da-saude-lanca-acao-nacional-de-combate-a-sifilis> Acesso em 09/12/2018

Cardoso, A. R. P., et al (2018). Análise dos casos de sífilis gestacional e congênita nos anos de 2008 a 2010 em Fortaleza, Ceará, Brasil. Ciência \& Saúde Coletiva, 23(2), 563-574. https://doi.org/10.1590/1413-81232018232.01772016

Cavalcante, A. N., Araújo, M. A. L., Nobre, M. A., \& Almeida, R. L. F. D. (2019). Fatores associados ao seguimento não adequa do de crianças com sífilis congênita. Revista de Saúde Pública, 53, 95..https://doi.org/10.11606/s1518-8787.2019053001284

Costa, C. C. da., Freitas, L. V., Sousa, D. M. do N., Oliveira, L. L. de., Chagas, A. C. M. A., Lopes, M. V. de O. \& Damasceno, A. K. de C. (2013). Sífilis congênita no Ceará: análise epidemiológica de uma década. Revista da Escola de Enfermagem da USP,47(1), 152-159. https://doi.org/10.1590/S008062342013000100019

De França, I. S. X., Batista, J. D. A. L., Coura, A. S., de Oliveira, C. F., Araújo, A. K. F., \& de Sousa, F. S. (2015). Fatores associados à notificação da sífilis congênita: um indicador de qualidade da assistência pré-natal. Revista da Rede de Enfermagem do Nordeste, 16(3), 374-381. 10.15253/21756783.2015000300010

De Macêdo, Vilma Costa de Lira, P. I. C., de Frias, P. G., Romaguera, L. M. D., Caires, S. D. F. F., \& de Alencar Ximenes, R. A. (2017). Fatores de risco para sífilis em mulheres: estudo caso-controle. Revista de Saúde Pública, 51, 1-12.https://dx.doi.org/10.11606/s1518-8787.2017051007066 
Research, Society and Development, v. 10, n. 3, e42410311568, 2021

(CC BY 4.0) | ISSN 2525-3409 | DOI: http://dx.doi.org/10.33448/rsd-v10i3.11568

Domingues, R. M. S. M., \& Leal, M. do C. (2016). Incidência de sífilis congênita e fatores associados à transmissão vertical da sífilis: dados do estudo Nascer no Brasil. Cadernos de Saúde Pública, 32(6), e00082415. https://dx.doi.org/10.1590/0102-311X00082415

Holztrattner, J. S., da Costa Linch, G. F., Paz, A. A., Gouveia, H. G., \& Coelho, D. F. (2019). Sífilis congênita: realização do pré-natal e tratamento da gestante e de seu parceiro. Cogitare Enfermagem, 24.

Lima, V. C., Mororó, R. M., Martins, M. A., Ribeiro, S. M. \& Linhares, M. S. C.(2017). Perfilepidemiológicodoscasosdesífiliscongênitaemummunicípiodemédio porte no nordeste brasileiro. J. Health Biol Sci;5(1):56-61. 10.12662/2317-3076jhbs.v5i1.1012.p.56-61.2017

Macêdo, V. C. de., Bezerra, A. F. B., Frias, P. G. D., \& Andrade, C. L. T. D. (2009). Avaliação das ações de prevenção da transmissão vertical do HIV e sífilis em maternidades públicas de quatro municípios do Nordeste brasileiro. Cadernos de Saúde Pública, 25(8), 1679-1692. https://dx.doi.org/10.1590/S0102$311 \times 2009000800004$

Oliveira, E. H., Silveira, J. A. V. da, Sampaio, S. S. de C., Verde, R. M. C. L., Soares, L. F., \& Costa, S. C. R. (2020). Análise dos casos notificados de sífilis na gestação no estado da Paraíba, Brasil. Research, Society and Development, 9(1), e179911900. https://doi.org/10.33448/rsd-v9i1.1900

Pires, A.C., Oliveira, D., Rocha, G., Santos, A. (2014). Ocorrência de sífilis congênita e os principais fatores relacionados aos índices de transmissão da doença no Brasil da atualidade - revisão de literatura.REVISTA UNINGÁ REVIEW, 19(1). http://revista.uninga.br/index.php/uningareviews/article/view/1522

Saraceni, V., Pereira, G. F. M., da Silveira, M. F., Araujo, M. A. L., \& Miranda, A. E. (2017). Vigilância epidemiológica da transmissão vertical da sífilis: dados de seis unidades federativas no Brasil. Revista Panamericana de Salud Pública, 41, e44. 10.26633/RPSP.2017.44

Vescovi, J. S., \&Schuelter-Trevisol, F. (2020). Increase of incidence of congenital syphilis in Santa Catarina State between 2007-2017: temporal trend analysis. Revista paulista de pediatria :orgao oficial da Sociedade de Pediatria de São Paulo, 38, e2018390. https://doi.org/10.1590/1984$0462 / 2020 / 38 / 2018390$ 